\title{
The Effectiveness of Nursing Delivered Interventions on Readmission Rate among Patients Post Coronary Artery Bypass Graft Surgery; a Systematic Review
}

\author{
Hayat S. Abu Shaikha1, Mohammad R. AlOsta ${ }^{2}$, Mohammad A. Abu Sabra ${ }^{3}$, Jafar A. Alshraideh \\ ${ }^{1}$ Lecturer, School of Nursing, Philadelphia University, Amman, Jordan, ${ }^{2}$ Lecturer, School of Nursing, the University \\ of Jordan, Amman, Jordan, ${ }^{3}$ Lecturer, School of Nursing, Al-Zaytoonah University of Jordan (ZUJ), Amman, \\ Jordan, ${ }^{4}$ Professor, School of Nursing, the University of Jordan, Amman, Jordan
}

\begin{abstract}
Introduction: Despite that hospital readmission after $\mathrm{CABG}$ surgery became an international concern, few studies have focused on nurse's role in the reduction of the readmission rate.The purpose: This review aims to assess the effectiveness of nursing interventions that are reported to reduce patient's readmission post CABG surgery. Methods: a systematic review following the PRISM Aprotocol was operated. Relevant studies were retrieved from two main electronic databases. Results: Eight studies met the inclusion criteria. The retrieved studies were conducted to identify the effectiveness of nursing interventions on readmission rate among patients post CABG surgery. Different types of nursing interventions were identified and generally, it decreased the readmission rate among patients in most of the studies. Conclusion: Different nursing interventions have been reported to reduce readmission rate after coronary artery bypass graft surgery. However, a better reporting of these nursing interventions and linking it with nursing staff training and qualification is recommended.
\end{abstract}

This review is registered at PROSPERO; the registration number is: CRD42020143206

Keywords: coronary artery graft, discharge education, nursing interventions, readmission

\section{Introduction}

Coronary Artery Bypass Graft (CABG) surgery is the first line treatment for multi-vessel coronary artery disease $^{[1]}$. Around 151474 patients underwent CABG surgery in the United States (US) during the year 2015 ${ }^{[2]}$. However; the readmission rate of CABG patients within 30 days after discharge is impressively high ${ }^{[3]}$. More specifically, they reported that 28,601 adult CABG patients out of $177,229(16 \%)$ in the US were readmitted within 30 days. Another study revealed that more than

\section{Correspondence author:}

Jafar A. Alshraideh, PhD

Professor, School of Nursing, the University of Jordan, Amman, Jordan, jalasad@ju.edu.jo,

Tel: 00962777484556

Fax: 00962-6-5300244
$60 \%$ of $\mathrm{CABG}$ patient readmissions occur within the first week post discharge ${ }^{[4]}$.

Decreasing readmissions post cardiac surgery has turn into a priority worldwide. Unclear discharge instructions and lack of follow up plans are among the reported reasons for hospital readmissions ${ }^{[5]}$. Patient experience with discharge instructions shows that patients who discharged with sufficient information and provided follow-up care were better in managing post-operative problems and experienced improved post discharge ${ }^{[6]}$.

Nurses play a major role in providing multidimensional interventions, for instance; support, training and education, and rehabilitation $)^{[7]}$. Examples of these interventions include home visits, rehabilitation programs, discharge education, telephone follow-up 
calls, telehealth, discharge planning, and discharges to short-term skilled nursing facilities help to reduce readmission rate ${ }^{[8]}$. A major intended outcome of these nursing delivered interventions is to reduce hospital readmission among CABG patients ${ }^{[9] \text {. }}$

The aim of this paper was to estimate the effectiveness of nursing interventions that are reported to reduce patient's readmission post CABG surgery.

\section{Materials and Methods}

Eligibility criteria and Information sources: Our literature review was managed by the PICOT system in order to develop a systematic search strategy. The components include interest population (P), interest question (I), interest comparison (C), interest outcome (O) and timeframe $(\mathrm{T})^{[10]}$. Our research centered on post CABG patients $(\mathrm{P})$ and the use of nursing procedures to decrease readmission (I). The interest comparison (C) was not related to our intent. With regard to the findings (O), it was hypothesized that the use of nursing administered therapies will minimize post-CABG surgery readmissions. We agreed on atimeframe (T) of all studies published from1999 to 2019.

The papers retrieved were included if they were: (1) clinical studies, literature review, or master's or doctoral thesis; (2) post coronary artery bypass graft surgery in adult patients; (3) nursing strategies aimed at preventing post-CABG readmission; (4) written in English and (5) published between 1999-2019. To find qualifying publications, two researchers searched two electronic databases: Medline (through PubMed) and CINAHL. The search started on June 20th, 2019 and ended on July 20th, 2019.

Search strategies: Through the search, Study records and systematic analyses have been identified. In order to include medical subject heading (MeSH) terminology, words were typically entered and extended where appropriate. primarily, large categories of search words, containing readmission, coronary artery graft, and nursing, were picked. To discover target studies, all available variations of the words for each class were then checked. In order to identify publications not found by electronic search, a manual search of the reference lists of qualifying studies was also performed.
Study selection:Separately, to choose studies that technically meet the inclusion criteria, two researchers checked headlines and abstracts of selected papers. After that, for eligibility, the full text of these studies was closely checked. Discrepancies were explained by discussions and by a third reviewer over the eligibility of studies.

Data items and Data collection process: The researchers carried out a systematic analysis of the included papers, extracted and compiled information from the studies in tables. A data extraction sheet was created by the researchers, and two reviewers extracted the data. Any inconsistencies were resolved, and information was included only if agreement was reached between the researchers.

Risk of bias in individual studies and Synthesis of results: The probability of bias in all studies was independently calculated by two researchers. The quantitative studies were analyzed using the checklist ${ }^{[10}$, ${ }^{11}$ of the Successful Public Health Practice Project with the Quality Assessment Method (QAT) (EPHPP). The retrieved systematic reviews were analyzed using the Essential Appraisals Skills Program (CASP) Checklist ${ }^{[12,}$ ${ }^{13}$. If the scores were in disagreement, by consulting a third reviewer, consensus was reached.Two researchers analyzed and synthesized information, by consensus, any difference between them was resolved, data was only included if both researchers agreed. It gave the facts a narrative-descriptive summary.

\section{Results}

Study Selection and Characteristics: An overall of (1208) papers was retrieved. After duplicates removal, (703) research paper titles and abstracts were screened. There were (72) related articles which were reviewed in full text, eight of which were deemed appropriate to be part of the analysis according to the Preferred Reporting Items for Systematic Reviews and Meta Analyses (PRISMA) protocol for systematic reviews ${ }^{\text {[14]. }}$

Eight studies, published between 1999 and 2018, were part of the review. There were $(n=5)$ experimental studies, $(n=2)$ review articles, and one observational retrospective study. Threearticles described the effect of more than one intervention on the readmission 
rate among $\mathrm{CABG}$ patients, and 5 studies reported the effect of only one intervention. Generally, the 8 articles reported interventions carried by nurses with divers nursing qualifications. One study reported the participation of cardiac surgery nurse practitioner, 2 reported advance practice nurses, 1 reported on trained research nurses, and 4 reported cardiac /coronary care nurses. A summary of studies characteristics is shown in table 1.

Table 1. Characteristics of literature included in review $(\mathbf{N}=\mathbf{8})$.

\begin{tabular}{|c|c|c|}
\hline & $\mathbf{N}(\%)$ & References (no.) \\
\hline \multicolumn{3}{|r|}{ Sample size } \\
\hline$<50$ & $2(25)$ & $\begin{array}{c}\text { (Barnason, Zimmerman, Nieveen, \& Hertzog, 2006; Fredericks \& } \\
\text { Yau, 2013) }\end{array}$ \\
\hline $50-200$ & $3(37.5)$ & $\begin{array}{c}\text { (Carroll, Rankin, \& Cooper, 2007; Naylor \& McCauley, 1999; } \\
\text { Negarandeh, Nayeri, Shirani, \& Janani, 2012) }\end{array}$ \\
\hline$>400$ & $1(12.5)$ & (Hall et al., 2014) \\
\hline \multicolumn{3}{|r|}{ Study design } \\
\hline Experimental & $5(62)$ & $\begin{array}{c}\text { (Carroll et al., 2007; Naylor \& McCauley, 1999; Negarandeh et al., } \\
\text { 2012) }\end{array}$ \\
\hline Observational & $1(13)$ & (Hall et al., 2014) \\
\hline Review & $2(25)$ & (Fredericks \& Yau, 2017; Mares \& McNally, 2013) \\
\hline \multicolumn{3}{|r|}{ Country } \\
\hline USA & $4(50)$ & $\begin{array}{c}\text { (Barnason et al., 2006; Carroll et al., 2007; Hall et al., 2014; Naylor } \\
\text { \& McCauley, 1999) }\end{array}$ \\
\hline Canada & $2(25)$ & (Fredericks \& Yau, 2013, 2017) \\
\hline Australia & $1(12.5)$ & (Mares \& McNally, 2013) \\
\hline Iran & $1(12.5)$ & (Negarandeh et al., 2012) \\
\hline
\end{tabular}

Risk of Bias within Studies: Reliable checklists were used to test the content of included papers. Using the QAT, the quality of quantitative studies was evaluated, which was interpreted by referring to the tool dictionary and a research analysis that analyzed components of quality evaluation and ratings for the EPHPP instrument ${ }^{[15]}$. In general, an appropriate consistency ranking was demonstrated by the research findings using quantitative designs (Supplemental Digital Content risk of bias in individual studies). Three out of 6 articles were rated as fair or good for selection bias monitoring, three articles used clinical trial design, and three were cohort studies. The confounding factors were discussed by just one article and two experiments were blinded. The reliability and validity of the instruments for collecting data were stated in most of the papers, and three articles discussed the withdrawal rate. On the other hand,the two reviews included showed a high-quality rating, only one of the studies failed to specify aresearch question. 


\section{Identification of commonly used interventions delivered by nurses}

Two out of 8 studies reported more than two interventions delivered by nurses. The first study ${ }^{[16]}$ examined the effect of a discharge planning program consist of multiple interventions delivered by Advance Practice Nurses (APNs). The program consists of a comprehensive discharge plan, followed by a 2 home visits within the first 10 days after discharge and a provision of a telephone call initiated on a weekly basis for 4 weeks. In the second study ${ }^{[17]}$, the researcher recruited coronary care nurses to implement the intervention. The intervention consists of a discharge plan initiated at admission and continued for 2 weeks after discharge by 2 home visits and provision of telephone numbers to enable patients to contact the nurses for any questions or clarification.

Two studies examined the effects of an individualized education delivered by nurses on the readmission rates among CABG patients. The first examined the effect of an individualized education delivered by a trained nurse via a telephone call on two points of time. Each call started with an identification of the learning needs followed by the educational material ${ }^{[18]}$. The second was a systematic review ${ }^{[19]}$ of 17 trials; 4 studies which examined the effect of individualized education on readmission rates among $\mathrm{CABG}$ patients. Nurses delivered the education in more than half of the trials (8/17). Of the 17 trials 10 reported that patients were given access to contact nurses who were responsible to answer questions and clarify issues related to the education.

One study ${ }^{[20]}$ reported the effect of home visits conducted by the same cardiac surgery nurse practitioner (NP) who was delivering care to the patient during inhospital period. The two visits took place within the first 10 days after discharge. During the visit, the NP was responsible to assessing the patient's recovery, providing necessary education, assessing adherence to medications and even changing the medication if required with coordination with the surgeon.
One study examined the effect of a tele-health technology delivered by nurses ${ }^{[21]}$ using a health communication intervention that utilizes a device called 'health buddy'. The nurse researcher prepared scripts including educational material related to the recovery of the CABG patients, recovery symptoms, strategies to manage symptoms, and reinforcement to enhance patient self-efficacy to manage their recovery. The study reported no direct contact between nurses and patients except through the device however; nurses were able to receive patient's questions through the health buddy and answer them.

Two studies reported the participation of nurses in cardiac rehabilitation programs after CABG surgery. The first study ${ }^{[22]}$ examined the effect of a shared intervention delivered by peer advisors (family or friend) and APNs. The intervention was built to enhance CABG patient's self-efficacy and ultimately increases their participation in the rehabilitation programs and reduces the readmission rates. The intervention starts in hospital before discharge and last for 12 weeks after discharge. Two APNs with aMaster degree and clinical experience in cardiac surgery nursing were responsible to recruit, train, and assign the peer advisors to the treatment group. The APNs provided home visits and at least 3 telephone calls to the patients, whereas the peer advisors called the patients on a weekly basis for 12 weeks. Moreover; the APN provided support to both patients and peer advisors.

The second study ${ }^{[23]}$ was a systematic review aimed to report the effectiveness of nurse-led cardiac rehabilitation program on readmission rates and other outcomes among CABG patients. The review included three trials however none of them examined the effectiveness of the nurse-led cardiac rehabilitation programs on readmission rates.A summary of reported interventions in each study is presented in table 2 . 
Subjectivity Study on Anxiety in Clinical Practice of Nursing Students

Table 2. General characteristics and factor weights of $P$ samples by type

\begin{tabular}{|c|c|c|c|}
\hline Type & ID & Factor weights & Religion \\
\hline \multirow{5}{*}{$\begin{array}{l}\text { Type1 } \\
(\mathrm{n}=5)\end{array}$} & $\mathrm{P}-1$ & 0.72 & Protestant \\
\hline & P-8 & 0.56 & None \\
\hline & $\mathrm{P}-11$ & 076 & None \\
\hline & $\mathrm{P}-14$ & 0.74 & Buddhism \\
\hline & P-16 & 0.68 & Buddhism \\
\hline \multirow{8}{*}{$\begin{array}{l}\text { Type2 } \\
(\mathrm{n}=8)\end{array}$} & $\mathrm{P}-3$ & -0.59 & Catholic \\
\hline & P-7 & 0.55 & Protestant \\
\hline & $\mathrm{P}-13$ & 0.63 & None \\
\hline & $\mathrm{P}-24$ & 0.61 & None \\
\hline & $\mathrm{P}-25$ & 0.45 & Buddhism \\
\hline & $\mathrm{P}-26$ & 0.67 & None \\
\hline & $\mathrm{P}-28$ & 0.78 & None \\
\hline & P-29 & 0.72 & None \\
\hline \multirow{4}{*}{$\begin{array}{l}\text { Type3 } \\
(\mathrm{n}=4)\end{array}$} & P-9 & 0.68 & Protestant \\
\hline & P-19 & 0.72 & None \\
\hline & $\mathrm{P}-21$ & 0.51 & Protestant \\
\hline & $\mathrm{P}-22$ & 0.80 & Catholic \\
\hline \multirow{7}{*}{$\begin{array}{l}\text { Type4 } \\
(\mathrm{n}=7)\end{array}$} & $\mathrm{P}-4$ & 0.60 & None \\
\hline & P-5 & 0.66 & None \\
\hline & P-6 & 0.50 & Buddhism \\
\hline & $\mathrm{P}-10$ & 0.59 & None \\
\hline & $\mathrm{P}-12$ & 0.60 & Protestant \\
\hline & $\mathrm{P}-15$ & 0.62 & None \\
\hline & P-18 & 0.59 & None \\
\hline
\end{tabular}

Evaluation of the effect of the interventions on the readmission rates

The eight studies presented a substantial variation in the length of follow up period and outcome measurement. Two studies evaluated the outcome 'readmission rate'at different time points; at 6 weeks, 3, 6, and 12 months $^{[22]}$, andat 1 week, 3 weeks, 8 weeks and 12 weeks ${ }^{[18]}$. Another two studies evaluated the outcome after 3 months of discharge $e^{[17,21]}$ and one study ${ }^{[20]}$. evaluated the outcome after 30 days. Naylor and McCauleystudy evaluated the outcome at 24 weeks ${ }^{[16]}$. The Fredericks and Yausy stematic review ${ }^{[19]}$ didn't specify any restrictions related to the follow-up periods and frequency of outcome measurements, whereas the Mares et al review didn't report a measurement of the outcome of concern ${ }^{[23]}$.
Overall; 4 studies showed a significant reduction in the readmission rate. The first study found zero readmission rates among the treatment group compared with 4 readmissions in the control group ${ }^{[18]}$. The second ${ }^{[19]}$ found that nurse delivered individualized education regardless of the number of sessions and delivery method, have significant effect on the reduction of the readmission rates after CABG surgery. The third study ${ }^{[16]}$ reported a significant reduction of the readmission rate after multiple interventions delivered by APNs at discharge and for 6 weeks after(12 readmission in the control group compared to 4 readmission in the treatment group; $p=0.02$ ) readmission remained significantly lower in the intervention group at 6 weeks and 24 weeks post discharge. The fourth study ${ }^{[20]}$ reported that home visits by cardiac surgery NPs resulted in a significant reduction in the readmission rate within 
30 days of discharge $(3.85 \%$ in the intervention group compared with $11.54 \%$ in the usual group $(\mathrm{N}=156, \mathrm{p}=$ $0.023)$.

Another three studies reported an insignificant reduction of the readmission rate among $\mathrm{CABG}$ patients after the delivery of multiple interventions developed and administered by nursing staff. The first study ${ }^{[17]}$ recruited coronary care nurses to administer the interventions. The authors reported no statistically significant reduction of the readmission rate $(p=0.5)$. Even though; the percentage of readmission rate in the intervention group was lower than the percentage in the control group (4.76 $\%$ and $14.63 \%$ respectively). The second study ${ }^{[21]}$ used telehealth technology, which showed no statistically significant reduction of the readmission rate(2 patients from the treatment group were readmitted compared with 1 patient from the control group). The third study ${ }^{[22]}$ showed no statistically significant difference in the readmission rates. Nevertheless; the trend of the readmission showed a fewer readmission between 3-6 months in the treatment group.

\section{Discussion}

Apparently; the review shows a scarcity of nursing studies that examine the effects of interventions delivered by nurses on the readmission rate of $\mathrm{CABG}$ patients. More than one third of the studies $(n=3,38 \%)$ reported that the intervention was delivered by nurses with advanced education; APNs and NPs ${ }^{[16,20]}$. Of these, $66.6 \%$ (two studies) reported that the APN and the NPs who delivered the interventions were the same nurses who cared for the patients during hospitalization period after the surgery. The continuity of care provided by the nurses appeared clearly in the statistically significant results on the outcome variables.

Two studies (25\%) examined the effect of remote education and follow-up of the patients through the use of technology (Health buddy) without any contact with the nurses except through the device ${ }^{[21]}$ or through the use of peer advisors with support from $\mathrm{APNs}^{[22]}$. The results of these studies showed insignificant results which might be explained by the partial or complete physical absence of the nurses during the delivery of the intervention.
Three studies reported the utilization of professional nurses with specialized training in cardiac intensive care or coronary care ${ }^{[17,18,23]}$ Whereas; $25 \%$ of the studies reported that the intervention delivered by professional nurses without any details if they have any other qualifications or training ${ }^{[19,21]}$

Our review limitations include constraints on English-language papers, a limited number of qualifying studies, and differences in the nature of the studies used.

\section{Conclusions}

This review gives an overview of nurses' interventions to reduce the readmission rate after CABG surgery. Our review revealed that; although nurses represent a significant part of healthcare providers in any health care organization and despite their role as health educators; the reporting of their participation continues to be limited. Most of the studies emphasize the intervention itself, regardless of the provider. This review has shown that the nurses as the providers of the intervention, their qualification or advanced education, their presence at the time of intervention and their familiarity with the patients while in-hospital has a significant effect on patients' outcome. The results of this review highlight the importance of reporting nurses' participation in improving CABG patient's outcomes after the discharge. We recommend that researchers (nurses or others)use the term"nurse" or "professional nurse" not health care provider or health educator when reporting on studies using nursing interventions to improve patients' outcomes and to report any advanced education or training of the nursing staff as this was found to have an effect on the results of the studies.

Ethical Statement: There were no necessary for ethics approvals

Funding Statement: none

Conflict of Interest Statement: No conflicts of interest exist.

\section{References}

1. Habib, R.H., et al., CABG versus PCI: greater benefit in long-term outcomes with multiple arterial bypass grafting. 2015. 66(13): p. 1417-1427. 
2. EJ, B.J.H.d. and s.s.-u.A.r.f.t.A.h.a. Circulation, Virani SS. Callaway CW. Chamberlain AM. Chang AR. Cheng S, et al. 2018. 137: p. 67-492.

3. Feng, T.R., et al., Coronary artery bypass graft readmission rates and risk factors-A retrospective cohort study. 2018. 54: p. 7-17.

4. Price, J.D., et al., Risk analysis for readmission after coronary artery bypass surgery: developing a strategy to reduce readmissions. 2013. 216(3): p. 412-419.

5. Amoah, D. and L.J.A.J.P.H.E. Mwanri, Determinants of hospital readmission of medical conditions in developing countries. 2016. 3(5): p. 1049.

6. Akbari, M., S.S.J.I.j.o.n. Celik, and m. research, The effects of discharge training and counseling on post-discharge problems in patients undergoing coronary artery bypass graft surgery. 2015. 20(4): p. 442.

7. Kadda, O., C. Marvaki, and D.J.H.s.j. Panagiotakos, The role of nursing education after a cardiac event. 2012. 6(4): p. 634.

8. Lazar, H.L.J.J.o.c.s., Reducing readmission risk following CABG surgery-Doing whatever it takes. 2018. 33(4): p. 171-171.

9. Mary, A.J.H.h.n., Prevention of 30-day readmission after coronary artery bypass surgery. 2017. 35(6): p. 326-334.

10. Riva, J.J., et al., What is your research question? An introduction to the PICOT format for clinicians. 2012. 56(3): p. 167.

11. Critical Appraisal Skills Programme - 2018 - CASP for Systematic Reviews(2).pdf $>$.

12. Effective Public Health Practice Project - 2003 Quality assessment tool for quantitative studies. pdf $>$.

13. Thomas et al. - 1998 - Dictionary for Quality Assessment Tool for Quantitative Studies (EPHPP) (2).pdf $>$.

14. Liberati, A., et al., G? tzsche PC, Ioannidis JP, Clarke M, Devereaux PJ, Kleijnen J, Moher D: The PRISMA statement for reporting systematic reviews and meta-analyses of studies that evaluate health care interventions: explanation and elaboration. 2009. 6(7): p. e1000100.

15. Thomas, B., et al., A process for systematically reviewing the literature: providing the research evidence for public health nursing interventions. 2004. 1(3): p. 176-184.

16. MD, N.J.J.o.C.N., McCauley KM. The effects of a discharge planning and home follow-up intervention on elders hospitalized with common medical and surgical cardiac conditions [see comments]. 1999. 14(1): p. 44-54.

17. Negarandeh, R., et al., The impact of discharge plan upon re-admission, satisfaction with nursing care and the ability to self-care for coronary artery bypass graft surgery patients. 2012. 11(4): p. 460465.

18. Fredericks, S. and T.J.W.j.o.n.r. Yau, Educational intervention reduces complications and rehospitalizations after heart surgery. 2013. 35(10): p. 1251-1265.

19. Fredericks, S. and T.J.I.j.o.n.s. Yau, Clinical effectiveness of individual patient education in heart surgery patients: a systematic review and meta-analysis. 2017. 65: p. 44-53.

20. Hall, M.H., et al., Cardiac surgery nurse practitioner home visits prevent coronary artery bypass graft readmissions. 2014. 97(5): p. 1488-1495.

21. Barnason, S., et al., Impact of a telehealth intervention to augment home health care on functional and recovery outcomes of elderly patients undergoing coronary artery bypass grafting. 2006. 35(4): p. 225-233.

22. Carroll, D.L., S.H. Rankin, and B.A.J.J.o.C.N. Cooper, The effects of a collaborative peer advisor/ advanced practice nurse intervention: cardiac rehabilitation participation and rehospitalization in older adults after a cardiac event. 2007. 22(4): p. 313-319.

23. Mares, M.A., S. McNally, and R.S.J.J.E.S. Fernandez, Effectiveness of nurse-led cardiac rehabilitation programs following coronary artery bypass graft surgery: a systematic review. 2018. 16(12): p. 2304-2329. 